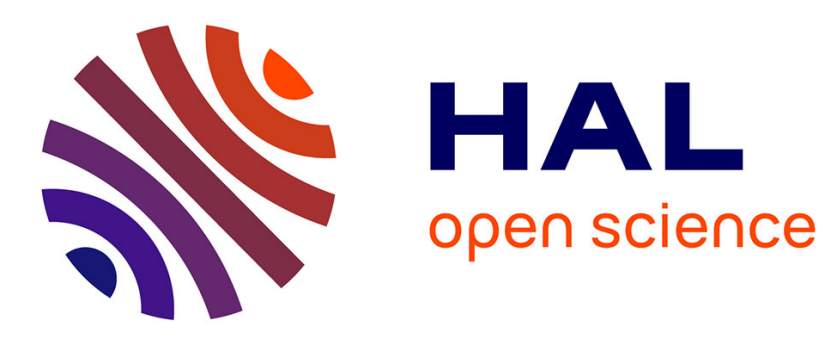

\title{
Méthode formelle de commande sous contraintes de temps dans les dioïdes
}

\author{
Saïd Amari, Isabel Demongodin, Jean Jacques Loiseau
}

\section{To cite this version:}

Saïd Amari, Isabel Demongodin, Jean Jacques Loiseau. Méthode formelle de commande sous contraintes de temps dans les dioïdes. Modélisation des Systèmes Réactifs, MSR'05, publié dans le Journal Européen des Systèmes Automatisés, RS série JESA, volume 39 - n 1-2-3/2005, Oct 2005, Grenoble, France. pp.319-334. hal-00362941

\section{HAL Id: hal-00362941 https://hal.science/hal-00362941}

Submitted on 19 Feb 2009

HAL is a multi-disciplinary open access archive for the deposit and dissemination of scientific research documents, whether they are published or not. The documents may come from teaching and research institutions in France or abroad, or from public or private research centers.
L'archive ouverte pluridisciplinaire HAL, est destinée au dépôt et à la diffusion de documents scientifiques de niveau recherche, publiés ou non, émanant des établissements d'enseignement et de recherche français ou étrangers, des laboratoires publics ou privés. 


\title{
Méthode formelle de commande sous contraintes de temps dans les dioïdes
}

\author{
Saïd Amari*- Isabel Demongodin**_ Jean Jacques Loiseau* \\ * IRCCyN, UMR CNRS 6597 \\ 1, rue de la Noë, BP 92 101, 44321 Nantes cedex 03, France. \\ \{said.amari, jean-jacques.loiseau\}@irccyn.ec-nantes.fr \\ ** LISA, FRE CNRS 2656 \\ 62 av. Notre-Dame-du-Lac 49000 Angers, France \\ isabel.demongodin@univ-angers.fr
}

\begin{abstract}
RESUME. Nous considérons dans ce papier, une classe de graphes d'événements temporisés contrôlés soumis à des contraintes temporelles. Ces graphes sont déterministes, dans le sens où leur comportement dépend seulement de leur marquage initial et des entrées de commande qu'on leur a appliquées. Le comportement de ces graphes peut être modélisé par des équations aux différences qui sont linéaires dans l'algèbre Min-Plus. Les contraintes temporelles sont traduites par des inéquations, qui sont également linéaires dans l'algèbre Min-Plus. Nous proposons alors une méthode pour la synthèse de commande qui garantit le respect des contraintes temporelles. Nous explicitons une loi de commande, qui, si deux conditions sont satisfaites, assure la validité des contraintes temporelles. La commande est un retour d'état causal impliquant des retards. La méthode est illustrée sur un exemple réel de processus de production.

ABSTRACT. We consider a class of controlled timed event graphs subject to strict temporal constraints. Such a graph is deterministic, in the sense that its behavior only depends on the initial marking and on the control that is applied. This behavior can be modelled by a system of difference equations that are linear in the Min-Plus algebra. The temporal constraint is represented by an inequation that is also linear in the Min-Plus algebra. Then, a method for the synthesis of a control law ensuring the respect of the constraint is described. We give explicit formulas characterizing a control law, which, if two conditions are satisfied, ensures the validity of the temporal constraints. It is a causal state feedback, involving delays. The method is illustrated on a real example of a production system.
\end{abstract}

Mots-CLES: Graphes d'événements temporisés, modèles Min-Plus linéaires, contraintes temporelles, commande.

KEYWORDS : Timed event graph, Min-Plus linear models, temporal constraint control. 


\section{Introduction}

Nous considérons dans cet article une classe de systèmes contrôlés déterministes soumis à des contraintes temporelles strictes. Les systèmes à temps critiques sont très fréquents dans le milieu industriel, par exemple, dans le cas des processus de traitement chimiques ou des procédés agroalimentaires. Les questions de vérification et de validation de propriétés temporelles ont été traitées par plusieurs auteurs, voir par exemple [ALU 94], [BER 91], [BON 01] et [GHE 91]. Dans ce travail, nous formulons la question en termes d'un problème de commande, nous calculons des entrées de commande pour que les contraintes temporelles soient respectées par le système commandé. Notre problème de commande est formulé en termes d'équations et d'inéquations dans l'algèbre Min-Plus. Les équations représentent le modèle du comportement du graphe d'événements temporisés et les inéquations expriment les contraintes temporelles qu'il faut respecter.

L'approche de commande à laquelle nous nous sommes intéressés est différente de la commande supervisée dans les réseaux de Petri ([HOL 97], [MOO 94]), puisque dans notre cas le temps est explicitement pris en compte. Notons qu'une étude sur la commande sous contrainte de temps a été développée dans [LAH 04]. Les auteurs ont considéré des graphes d'événements temporisés et le formalisme des dioïdes [BAC 92]. Ils ont défini un algorithme qui calcule un modèle de référence intégrant la contrainte temporelle, et ont ensuite proposé un correcteur pour que le système corrigé atteigne le modèle de référence. Ils ont limité leur étude à des structures de graphes d'événements temporisés soumis à une unique contrainte temporelle. Les auteurs s'intéressent plus précisément à des questions de rejet de perturbations et de poursuite de modèle et la contrainte temporelle est apparue comme une condition supplémentaire. Dans [AMA 04], le formalisme utilisé est aussi celui des graphes d'événements temporisés et des modèles linéaires dans l'algèbre des dioïdes.

Dans ce papier, nous proposons une méthode de synthèse de commande intégrant des retards sur les lois de commande et qui respecte plusieurs contraintes temporelles sur l'état du système commandé. La loi de commande est ainsi définie par une équation aux différences Min-Plus linéaire. Une première approche vers cette méthode de synthèse a été présentée dans [AMA 05]. La présente contribution est une généralisation pour les graphes d'événements 
temporisés soumis à plusieurs contraintes de temps, et à plusieurs entrées de commande.

Ce papier est structuré de la manière suivante. Dans la section 2, quelques définitions de base sont rappelées, notamment des notations concernant le dioïde Min-Plus, les graphes d'événements temporisés et leurs modèles Min-Plus linéaires ainsi que le concept de l'équation d'état. Le problème de trouver une commande causale qui vérifie les contraintes temporelles est formulé dans la section 3, et nous proposons dans la section 4 une procédure de synthèse de commande. Initialement, nous considérons le cas d'une seule contrainte temporelle et d'une seule entrée de commande. Deux conditions suffisantes sont proposées pour assurer l'existence d'une solution. Ensuite, nous généralisons la méthode aux cas de plusieurs contraintes différentes et de plusieurs entrées de commande. Finalement, la section 5 est consacrée à un exemple illustratif d'un processus réel de production.

\section{Outils de base}

\subsection{Algèbre des dioïdes}

Commençons par présenter quelques concepts généraux et notations qui seront utilisés dans la suite de ce papier. Un monoïde, noté $(D, \oplus)$, est un ensemble $D$, muni d'une loi de composition interne $\oplus$, qui est associative et possède un élément neutre, noté $\varepsilon: \forall a \in D, a \oplus \varepsilon=\varepsilon \oplus a=a$. Le monoïde est dit commutatif si la loi $\oplus$ est commutative. Un demi-anneau est un monoïde commutatif muni d'une deuxième loi, notée $\otimes$, qui est associative, distributive à droite et à gauche par rapport à la loi $\oplus$. Il admet un élément neutre, noté $e$, et un élément absorbant $\varepsilon$ : $\forall a \in D, a \otimes \varepsilon=\varepsilon \otimes a=\varepsilon$. Finalement, un dioïde est un demi-anneau pour lequel la loi $\oplus$ idempotente : $\forall a \in D, a \oplus a=a$. Le diö̈de est dit commutatif si la deuxième loi $\otimes$ est commutative. Nous considérons dans la suite, le diö̈de commutatif MinPlus, aussi appelé algèbre Min-plus et noté $(\mathbb{R} \cup\{-\infty\} \cup\{+\infty\}, \min ,+)$ ou $\overline{\mathbb{R}}_{\text {min }}$. Dans ce dioïde, la loi $\oplus$ correspond à l'application min, dont élément neutre est $\varepsilon=+\infty$, et la deuxième loi $\otimes$, correspond à la somme usuelle, avec comme élément neutre $e=0$. Notons toutefois que : $\varepsilon \otimes(-\infty)=(-\infty)+(+\infty)=\varepsilon=(+\infty)$.

Si $n \in \mathbb{N}$ et $v, w \in \overline{\mathbb{R}}_{\text {min }}^{n}$, on note $v \oplus w$, le vecteur dont les composantes $v_{i} \oplus \underline{w}_{i}:=\min \left(v_{i}, w_{i}\right)$, pour $i$ allant de 1 à $n$. Les nombres $p, n, q \in \mathbb{N}$ et les matrices $P \in \overline{\mathbb{R}}_{\text {min }}^{p \times n}, Q \in \overline{\mathbb{R}}_{\text {min }}^{n \times q}$ étant données, alors le produit matriciel dans $\overline{\mathbb{R}}_{\text {min }}$, noté $P . Q$, est défini comme suit :

$$
(P . Q)_{i j}=\bigoplus_{k=1}^{n}\left(P_{i k} \otimes Q_{k j}\right):=\min _{k}\left(P_{i k}+Q_{k j}\right)
$$

L'étoile de Kleene d'une matrice carré $M \in \overline{\mathbb{R}}_{\text {min }}^{n \times n}$ noté $M^{*}$, est défini comme suit : 


$$
M^{*}=\bigoplus_{i \in \mathbb{N}} M^{i} .
$$

La matrice $M^{0}$, notée $I_{n}$ est appelée matrice unité, dont les termes valent $e$ sur la diagonale et $\varepsilon$ ailleurs. Il est bien montré que, pour $v \in \mathbb{R}_{\min }^{n}, x=M^{*} v$ est la solution maximale de l'inéquation $x \leq M . x \oplus v$ et aussi de l'équation $x=M \cdot x \oplus v$.

\subsection{Graphes d'événements temporisés et modèles Min-Plus linéaires}

Un graphe d'événements est un réseau de Petri ordinaire où chaque place a exactement une transition en amont et une transition en aval. Un graphe d'événement temporisé est obtenu lorsque on associe des temporisations aux places ou aux transitions du graphe d'événement. Dans ce qui suit, on considère des graphes d'événements P-temporisés, qui ne sont temporisés que sur les places. Nous allons utiliser ces notations dans la suite du papier. On note par $P$, l'ensemble des places du graphe considéré, et par $T$ l'ensemble des transitions. Le nombre de transitions ayant des places en amont et en aval est noté $n$, et $m$ désigne le nombre de transitions sources qui n'ont pas de place en amont. Pour $t_{i}, t_{j} \in T$, on suppose que la place qui relie les transitions $t_{j}$ et $t_{i}$, est notée $p_{i j}$, est unique. Si elle existe, la temporisation correspondante est notée $\tau_{i j}$ et le marquage initial de cette place est noté $m_{i j}$. La plus grande temporisation du graphe d'événements considéré est $\tau^{\max }$

$$
\tau^{\max }=\max _{i j / p_{i j} \in P}\left\{\tau_{i j}\right\} .
$$

Un chemin $\alpha$ de la transition $t_{i}$ à la transition $t_{j}$ est la séquence de transitions et de places, $\left(t_{i}, p_{i k_{1}}, t_{k_{1}}, p_{k_{1} k_{2}}, t_{k_{2}}, \ldots \ldots . ., t_{j}\right)$. On note par $\tau_{\alpha}$ la somme des temporisations le long du chemin $\alpha$, qui est donnée par

$$
\tau_{\alpha}=\sum_{p_{u} \in \alpha} \tau_{k l} .
$$

On associe à chaque transition du graphe d'événements considéré une fonction du temps $t$, correspondant au nombre cumulé de franchissement de la transition à l'instant $t$. Cette fonction est appelée compteur. Les compteurs correspondants aux transitions sources sont les composantes du vecteur $u(t) \in \overline{\mathbb{R}}_{\text {min }}^{m}$, et les compteurs des autres transitions composent le vecteur $\theta(t) \in \overline{\mathbb{R}}_{\text {min }}^{n}$. Il est bien montré dans [Bac 92], que le comportement dynamique d'un graphe d'événements temporisé peut s'exprimer sous forme d'une équation linéaire dans $\overline{\mathbb{R}}_{\text {min }}$, elle est donnée par

$$
\theta(t)=\overbrace{\tau=0}^{\tau_{\max }}\left(A_{\tau} \cdot \theta(t-\tau) \oplus B_{\tau} \cdot u(t-\tau)\right),
$$

où $A_{\tau} \in \overline{\mathbb{R}}_{\min }^{n \times n}$ est la matrice dont le terme $A_{\tau, i j}$ égal à $m_{i j}$, le nombre de jetons initiaux dans la place $p_{i j}$, si cette place existe et la temporisation associée est $\tau$, et $\varepsilon$ sinon. Egalement les termes des matrices $B_{\tau} \in \overline{\mathbb{R}}_{\min }^{n \times m}$ correspondent aux 
marquages initiaux des places de sortie des transitions sources. L'équation (1) est implicite en général. Elle est souvent remplacée par sa solution explicite suivante,

$$
\theta(t)=\underset{\tau>0}{\oplus}\left(A_{0}^{*} \cdot A_{\tau} \cdot \theta(t-\tau) \oplus A_{0}^{*} \cdot B_{\tau} \cdot u(t-\tau)\right),
$$

où $A_{0}^{*}$ est l'étoile de Kleene de la matrice $A_{0}$ définie précédemment dans cette section.

\subsection{Equation d'état}

Nous considérons par la suite quelques hypothèses qui seront nécessaires dans notre étude.

$\left(H_{1}\right)$ Toutes les temporisations du graphe sont commensurables à 1.

Par analogie avec la théorie des systèmes linéaires classiques, l'équation explicite (2) peut se transformer à une forme d'équation d'état. Pour obtenir un modèle d'état, nous décomposons toutes les places temporisées à $\tau>1$ en $\tau$ places temporisées à 1 , et nous ajoutons $(\tau-1)$ transitions intermédiaires. Nous avons associé des compteurs à ces transitions intermédiaires, qui sont les composantes d'un vecteur $\bar{\theta}(t) \in \overline{\mathbb{R}}_{\text {min }}^{n^{\prime}}$, et nous notons par $x(t)$ le vecteur d'état résultant

qui appartient à $\overline{\mathbb{R}}_{\min }^{N}$, avec $N=n+n^{\prime}$.

$$
x(t)=\left[\begin{array}{l}
\theta(t) \\
\bar{\theta}(t)
\end{array}\right],
$$

La commande agit sans retard, c'est-à-dire que toutes les temporisations des places qui ont des transitions sources en amont, sont nulles.

Cette hypothèse n'est pas limitative, car on peut toujours étendre le graphe. On ajoute des places et des transitions intermédiaires pour que le graphe résultant satisfasse cette supposition. Le comportement dynamique du graphe d'événements temporisé étendu est décrit par une équation de la forme

$$
x(t)=\hat{A}_{0} \cdot x(t) \oplus \hat{A}_{1} \cdot x(t-1) \oplus \hat{B} \cdot u(t),
$$

qui peut s'écrire sous une forme explicite

$$
x(t)=A \cdot x(t-1) \oplus B . u(t),
$$

avec $A=\hat{A}_{0}^{*} \cdot \hat{A}_{1}$ et $B=\hat{A}_{0}^{*} \cdot \hat{B}$.

Toutes ces équations et notations permettent de montrer que le comportement du graphe d'événements temporisé commandé est déterministe, dépend de l'entrée $u(t)$ et toutes les conditions initiales. L'équation d'état (3) est équivalente à la formulation suivante :

pour tout $\tau$ entier tel que $\tau \geq 1$.

$$
x(t)=A^{\tau} \cdot x(t-\tau) \oplus\left[\bigoplus_{k=0}^{\tau-1} A^{k} \cdot B \cdot u(t-k)\right],
$$


Dans la suite du travail, nous supposons que l'entrée $u(t)$ est réellement la commande, qui peut être donnée arbitrairement. Par exemple dans les processus de production, l'entrée peut correspondre à une autorisation d'exécution d'une opération. Le début d'une tâche exécutée par un robot, par exemple, peut se représenter par une telle entrée de commande.

$\mathrm{Au}$ départ, nous nous limiterons dans notre étude à une seule entrée de commande, ensuite nous s'intéresserons aux cas plusieurs entrées.

Nous considérons une seule entrée de commande, c'est-à-dire $m=1$.

La matrice $B$ devient un vecteur colonne dans ce cas. Donc, juste une seule transition est contrôlable, notée $t_{u}$, ça signifie que tous les éléments de la matrice sont égaux à $\varepsilon$, sauf $B_{1}$.

\subsection{Exemple}

Nous considérons le graphe d'événements temporisé de la Figure 1. Pour ce graphe, l'équation (1) donne

$$
\theta(t)=A_{0} \theta(t) \oplus A_{1} \theta(t-1) \oplus A_{2} \theta(t-2) \oplus A_{4} \theta(t-4) \oplus B u(t),
$$

où $A_{0}=\left(\begin{array}{lll}\varepsilon & 2 & 2 \\ \varepsilon & \varepsilon & \varepsilon \\ \varepsilon & \varepsilon & \varepsilon\end{array}\right), A_{1}=\left(\begin{array}{lll}\varepsilon & \varepsilon & \varepsilon \\ \varepsilon & \varepsilon & 1 \\ \varepsilon & \varepsilon & \varepsilon\end{array}\right), A_{2}=\left(\begin{array}{lll}\varepsilon & \varepsilon & \varepsilon \\ e & \varepsilon & \varepsilon \\ \varepsilon & \varepsilon & \varepsilon\end{array}\right), A_{4}=\left(\begin{array}{lll}\varepsilon & \varepsilon & \varepsilon \\ \varepsilon & \varepsilon & \varepsilon \\ \varepsilon & e & \varepsilon\end{array}\right), B=\left(\begin{array}{l}e \\ \varepsilon \\ \varepsilon\end{array}\right)$.

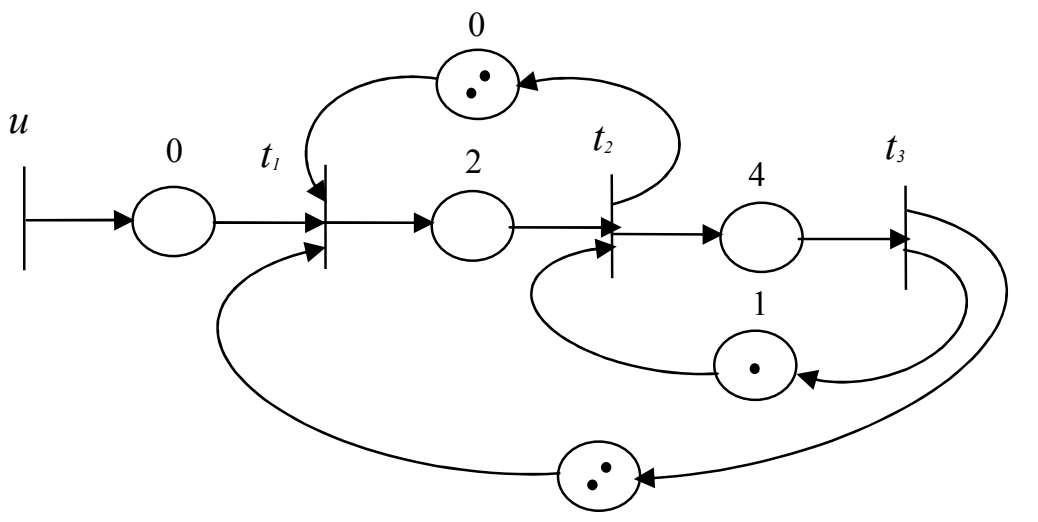

Figure 1: Graphe d'événements P-temporisé 




Figure 2 : Graphe équivalent étendu

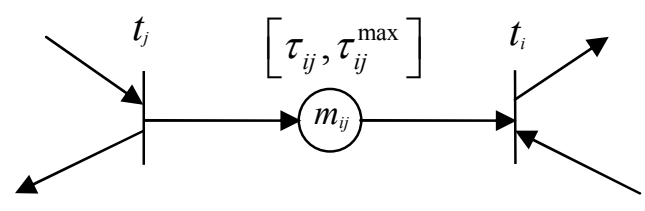

Figure 3 : Contrainte temporelle

L'équation explicite 2 est donnée par

$$
\theta(t)=\left(\begin{array}{lll}
\varepsilon & \varepsilon & 3 \\
\varepsilon & \varepsilon & 1 \\
\varepsilon & \varepsilon & \varepsilon
\end{array}\right) \theta(t-1) \oplus\left(\begin{array}{lll}
2 & \varepsilon & \varepsilon \\
e & \varepsilon & \varepsilon \\
\varepsilon & \varepsilon & \varepsilon
\end{array}\right) \theta(t-2) \oplus\left(\begin{array}{lll}
\varepsilon & 2 & \varepsilon \\
\varepsilon & \varepsilon & \varepsilon \\
\varepsilon & e & \varepsilon
\end{array}\right) \theta(t-4) \oplus\left(\begin{array}{l}
e \\
\varepsilon \\
\varepsilon
\end{array}\right) u(t)
$$

Nous avons étendu le graphe initial pour avoir un nouveau graphe équivalent avec des temporisations égales à 1 ou 0 . Nous obtenons le graphe de la Figure 2. Alors, l'équation d'état résultante est donnée par

$$
x(t)=\left(\begin{array}{lllllll}
\varepsilon & \varepsilon & 3 & 2 & \varepsilon & \varepsilon & 2 \\
\varepsilon & \varepsilon & 1 & e & \varepsilon & \varepsilon & \varepsilon \\
\varepsilon & \varepsilon & \varepsilon & \varepsilon & \varepsilon & \varepsilon & e \\
e & \varepsilon & \varepsilon & \varepsilon & \varepsilon & \varepsilon & \varepsilon \\
\varepsilon & e & \varepsilon & \varepsilon & \varepsilon & \varepsilon & \varepsilon \\
\varepsilon & \varepsilon & \varepsilon & \varepsilon & e & \varepsilon & \varepsilon \\
\varepsilon & \varepsilon & \varepsilon & \varepsilon & \varepsilon & e & \varepsilon
\end{array}\right) x(t-1) \oplus\left(\begin{array}{l}
e \\
\varepsilon \\
\varepsilon \\
\varepsilon \\
\varepsilon \\
\varepsilon \\
\varepsilon
\end{array}\right) u(t) .
$$

\section{Formulation du problème}

Les problèmes de contraintes de temps strict sont très souvent rencontrés dans les processus industriels. On peut considérer comme exemple un système de 
production qui comporte un four pour réaliser un traitement thermique. La durée des traitements thermiques est fixée, ou définie par un intervalle de temps. On a besoin alors de contrôler le système pour respecter cette contrainte. D'après la définition des graphes d'événements temporisés, la temporisation de chaque place, correspond à la durée minimale de séjour de jetons dans cette place. Une durée maximale apparaît comme une contrainte supplémentaire qui devrait être vérifiée. Plutôt qu'un problème de vérification, nous allons formuler la question comme un problème de commande qui consiste à calculer en ligne le paramètre $u(t)$ de façon à assurer que la contrainte temporelle est bien satisfaite.

On considère $p_{i j}$ la place soumise à la contrainte stricte. Un intervalle de temps $\left[\tau_{i j}^{\min }, \tau_{i j}^{\max }\right]$ est associé à cette place où se situe la contrainte temporelle, avec ${ }_{i j}^{\min }=\tau_{i j}$ (voir Figure 3). Cette contrainte s'exprime par cette inéquation suivante,

$$
m_{i j} \cdot x_{j}\left(t-\tau_{i j}\right) \geq x_{i}(t) \geq m_{i j} \cdot x_{j}\left(t-\tau_{i j}^{\max }\right)
$$

où le produit est défini dans $\overline{\mathbb{R}}_{\min }, m_{i j}$ est le marquage initial de la place $p_{i j}$. L'inéquation de gauche est satisfaite en tenant compte du modèle linéaire (3). La deuxième qui est donnée par

$$
x_{i}(t) \geq m_{i j} x_{j}\left(t-\tau_{i j}^{\max }\right),
$$

dont le produit est dans $\overline{\mathbb{R}}_{\text {min }}$, est en réalité la contrainte supplémentaire à satisfaire.

Nous souhaitons déterminer des commandes $u(t)$ pour garantir le respect de (5) pour $t>1$. Nous recherchons des lois de commande bien posée, sous la forme d'un retour d'état (feedback) causal, qui sont donnés comme suit :

$$
u(t)=F . x(t-1),
$$

pour $t>1$, où le produit est au sens de l'algèbre Min-Plus, et $F \in \overline{\mathbb{R}}_{\min }^{m \times N}$.

\section{Synthèse de commande}

\subsection{Une seule contrainte et une seule commande}

Nous proposons une méthode de synthèse de commande pour résoudre notre problème, pour cela, nous supposons que l'hypothèse supplémentaire suivante est satisfaite.

$\left(H_{2}\right)$ Il existe un chemin $\alpha$ qui relie la transition $t_{u}$ et $t_{j}$. 
Nous notons par $\tau_{\alpha}$ la somme des temporisations de toutes les places qui appartiennent à ce chemin. Nous remplaçons $\tau$ par $\tau_{\alpha}$ dans l'équation (4), et d'après la définition de $t_{u}$, nous avons

$$
x_{j}(t) \leq\left(A^{\tau_{\alpha}}\right)_{j u} u\left(t-\tau_{\alpha}\right) .
$$

Nous appliquons à nouveau l'équation (4) avec $\tau:=\phi$, nous obtenons l'expression explicite suivante de $x_{i}(t)$

pour chaque entier $\phi \geq 1$.

$$
x_{i}(t)=\bigoplus_{r=1}^{N}\left(A^{\phi}\right)_{i r} x_{r}(t-\phi) \oplus\left[\bigoplus_{k=0}^{\phi-1}\left(A^{k} B\right)_{i} u(t-k)\right],
$$

Théorème 1 Prenant $\phi=\tau_{i j}^{\max }+\tau_{\alpha}+1$, l'inéquation

$$
u(t) \leq \underset{r=1}{\oplus}\left[\left(\left(A^{\phi}\right)_{i r}-\left(A^{\tau_{\alpha}}\right)_{j u}-m_{i j}\right) x_{r}(t-1)\right],
$$

définit des commandes causales qui garantissent le respect de la contrainte (5) si les deux conditions suivantes sont satisfaites :

(i) $\left(A^{\phi}\right)_{i r} \geq\left(A^{\tau_{\alpha}}\right)_{j u} \cdot m_{i j}, \quad$ pour $r$ de 1 à $N$,

ii) $\left(A^{k} B\right)_{i} \geq\left(A^{\tau_{\alpha}}\right)_{j u} \cdot m_{i j}, \quad$ pour $k=0$ à $\phi-1$.

Démonstration Compte tenu de (7), il apparaît que la contrainte (5) est satisfaite si les deux conditions suivantes sont vérifiées

$$
\bigoplus_{r=1}^{N}\left(A^{\phi}\right)_{i r} x_{r}\left(t-\phi+\tau_{i j}^{\max }\right) \geq m_{i j} x_{j}(t),
$$

et

$$
\bigoplus_{k=0}^{\phi-1}\left(A^{k} B\right)_{i} u\left(t-k+\tau_{i j}^{\max }\right) \geq m_{i j} x_{j}(t)
$$

Ensuite, en tenant compte de (6), ces conditions deviennent

et

$$
\begin{aligned}
& \bigoplus_{r=1}^{N}\left(\left(A^{\phi}\right)_{i r}-m_{i j}\right) x_{r}\left(t-\phi+\tau_{i j}^{\max }\right) \geq\left(A^{\tau_{\alpha}}\right)_{j u} u\left(t-\tau_{\alpha}\right), \\
& \bigoplus_{k=0}^{\phi-1}\left(\left(A^{k} B\right)_{i}-m_{i j}\right) u\left(t-k+\tau_{i j}^{\max }\right) \geq\left(A^{\tau_{\alpha}}\right)_{j u} u\left(t-\tau_{\alpha}\right) .
\end{aligned}
$$

Choisissons $\phi=\tau_{i j}^{\max }+\tau_{\alpha}+1$ comme dans le théorème, les conditions (i) et (ii) sont satisfaites, et la loi de commande satisfait l'inéquation du théorème, alors on peut vérifier facilement que la contrainte (5) est bien respectée.

Corollaire $1 \mathrm{Il}$ existe toujours une loi de commande causale qui satisfait la contrainte (5), si les marquages initiaux de la place $p_{i j}$ et les places du chemin $\alpha$ de $t_{u}$ à $t_{j}$ sont nuls. Cette loi de commande est donnée par

$$
u(t)=\bigoplus_{r=1}^{N}\left(A^{\phi}\right)_{i r} x_{r}(t-1) .
$$


Démonstration Dans ce cas les deux conditions du théorème se traduisent par

(i) $\left(A^{\phi}\right)_{i r} \geq e$, pour $r$ de 1 à $N$,

(ii) $\left(A^{k} B\right)_{i} \geq e$ pour $k=0$ à $\phi-1$.

Ces conditions sont satisfaites trivialement, on montre ainsi que la loi de commande résultante est celle donnée dans le corollaire 1.

Exemple Nous revenons à l'exemple de la remarque 1. Dans ce cas, la temporisation maximale associée à la place $p_{i j}$ est $\tau_{i j}^{\max }=\tau_{21}^{\max }=3$ unités de temps, et la temporisation minimale égale à 2 . Cette contrainte temporelle correspond à l'inéquation (8).

$$
x_{2}(t) \geq x_{1}(t-3) .
$$

On vérifie d'abord que l'hypothèse $\left(\mathrm{H}_{2}\right)$ est bien vérifiée. On voit bien qu'il existe un chemin de $t_{u}$ à $t_{j}$, c'est-à-dire de $t_{u}$ à $t_{1}$. La temporisation de ce chemin est $\tau_{\alpha}=e$. Dans ce cas, on peut écrire l'équation (4) comme suit :

avec

$$
x(t)=A^{4} x(t-4) \oplus\left[\bigoplus_{k=0}^{3} A^{k} B u(t-k)\right],
$$

$$
A^{4}=\left(\begin{array}{lllllll}
4 & 2 & \varepsilon & \varepsilon & 3 & 4 & 5 \\
2 & \varepsilon & \varepsilon & \varepsilon & 1 & 2 & \varepsilon \\
\varepsilon & e & \varepsilon & \varepsilon & \varepsilon & \varepsilon & \varepsilon \\
\varepsilon & \varepsilon & 4 & 5 & 2 & 3 & 4 \\
\varepsilon & \varepsilon & 3 & 2 & \varepsilon & 1 & 2 \\
e & \varepsilon & \varepsilon & \varepsilon & \varepsilon & \varepsilon & 1 \\
\varepsilon & \varepsilon & 1 & e & \varepsilon & \varepsilon & \varepsilon
\end{array}\right) \quad \text { et } \quad B=\left(\begin{array}{c}
e \\
\varepsilon \\
\varepsilon \\
\varepsilon \\
\varepsilon \\
\varepsilon \\
\varepsilon
\end{array}\right) .
$$

D'après le corollaire 1 , les conditions du théorème 1 sont vérifiées, et la loi de commande qui garantit le respect de la contrainte temporelle (8) s'exprime par

$$
u(t)=\bigoplus_{r=1}^{7}\left(A^{4}\right)_{2 r} x_{r}(t-1)=2 x_{1}(t-1) \oplus 1 x_{5}(t-1) \oplus 2 x_{6}(t-1),
$$

Toutes les lois de commande inférieure à celle qu'on a calculé, garantissent le respect de contrainte.

\subsection{Plusieurs contraintes et une seule commande}

Nous nous intéressons de nouveau à des graphes d'événements temporisés, avec une seule transition source, qui est l'unique commande du graphe. Nous considérons 
$Z$ places soumises à des contraintes supplémentaires de temps, notées $p_{z}$, pour $z=1$ à $Z$. Pour chaque place contrainte $p_{z}$, on note respectivement par $m_{z}, \tau_{z}$ et $\tau_{z}^{\max }$, le marquage initial, la temporisation minimale et la temporisation maximale associées à cette place. Les transitions $t_{z}$ et $t_{z^{\prime}}$ représentent les transitions d'entrées et de sortie de la place $p_{z}$ et les variables $x_{z}$ et $x_{z^{\prime}}$ défissent les compteurs correspondants à ces transitions. Nous notons par $\lambda_{z}$ la somme des temporisation le long du chemin allant de $t_{u}$ à $t_{z}$. Les contraintes temporelles sont exprimées par les inéquations suivantes :

$$
m_{z} x_{z}\left(t-\tau_{z}\right) \geq x_{z^{\prime}}(t) \geq m_{z} x_{z}\left(t-\tau_{z}^{\max }\right)
$$

pour $z$ allant de1 à $Z$.

Nous notons par $u_{z}(t)$ la loi de commande calculée par la méthode précédente pour satisfaire la contrainte temporelle (9).

Théorème 2 L'équation

$$
u(t)=\bigoplus_{z=1}^{Z} u_{z}(t)
$$

avec $u_{z}(t)=\oplus_{=1}^{N}\left[\left(\left(A^{\phi_{z}}\right)_{z^{\prime} r}-\left(A^{\tau_{z}}\right)_{z u}-m_{z}\right) x_{r}(t-1)\right]$, et $\phi_{z}=\tau_{z}^{\max }+\tau_{z}+1$, pour $z=1$ à $Z$, déf́linit une commande causale qui respecte toutes les contraintes temporelle (9), si les conditions suffisantes suivantes sont toutes satisfaites, pour $z=1$ à $Z$.

(iii) $\left(A^{\phi_{z}}\right)_{z^{\prime} r} \geq\left(A^{\tau_{z}}\right)_{z u} \cdot m_{z} \quad$ pour $\quad r=1$ à $N$,

(iv) $\left(A^{k} B\right)_{z^{\prime}} \geq\left(A^{\tau_{z}}\right)_{z u} \cdot m_{z} \quad$ pour $k=0$ à $\phi_{z}-1$.

Démonstration D'après le théorème 1 , la loi de commande $u_{z}(t)$ valide la $z^{\text {éme }}$ contrainte, si les conditions (iii) et (iv) du théorème sont satisfaites.

Nous avons, pour $z=1$ à $Z$,

$$
u_{z}(t) \geq \underset{z=1}{Z} u_{z}(t)
$$

Finalement, il est clair que la commande $u(t)=\oplus_{z=1}^{Z} u_{z}(t)$ satisfait toutes les $Z$ contraintes temporelles.

\subsection{Plusieurs contraintes et plusieurs commandes}

Dans cette section, nous considérons des graphes d'événements temporisés avec plusieurs transitions sources, dont les compteurs associés constituent autant d'entrées de commande. Nous supposons dans un premier temps que $p_{i j}$ est l'unique place soumise à une contrainte temporelle supplémentaire. Nous souhaitons alors calculer un vecteur de commande $u(t) \in \overline{\mathbb{R}}_{\text {min }}^{m}$, avec $m \geq 1$, qui va vérifier la contrainte (5). Pour chaque transition source, nous allons associer une variable 
compteur, notée $u_{s}(t)$ pour $s=1$ à $m$, et nous notons aussi par $\lambda_{s}$, la somme des temporisations le long du chemin allant de $t_{u_{s}}$ à $t_{j}$.

Théorème 3 Le respect de la contrainte temporelle (5) est garanti si il existe un entier $s$ tel que :

$$
u_{s}(t) \leq \oplus_{r=1}^{N}\left[\left(\left(A^{\phi_{s}}\right)_{i r}-\left(A^{\lambda_{s}} B\right)_{j s}-m_{i j}\right) x_{r}(t-1)\right],
$$

avec $\phi_{s}=\tau_{i j}^{\max }+\lambda_{s}+1, \quad u_{i^{\prime}}(t)=\varepsilon$ pour $i^{\prime} \neq s$ et les conditions suivantes sont satisfaites,

(v) $\left(A^{\phi_{s}}\right)_{i r} \geq\left(A^{\lambda_{s}} B\right)_{j s} \cdot m_{i j} \quad$ pour $r=1$ à $N$,

(vi) $\left(A^{k} B\right)_{i s} \geq\left(A^{\lambda_{s}} B\right)_{j s} \cdot m_{i j} \quad$ pour $k=0$ à $\left(\phi_{s}-1\right)$.

Démonstration Nous remplaçons $\tau$ par $\lambda_{s}$ dans l'équation (4), et d'après la fonction compteur de la transition $t_{j}$ et du fait qu'il existe un chemin qui relie une composante d'indice $s$ du vecteur de commande et $t_{j}$, nous avons

$$
x_{j}(t) \leq\left(A^{\lambda_{s}} B\right)_{j s} u_{s}\left(t-\lambda_{s}\right) .
$$

Nous appliquons à nouveau la formulation (4) pour $\tau:=\phi_{s}$,

$$
\left.x(t)=A^{\phi_{s}} x\left(t-\phi_{s}\right) \oplus\left[\underset{k=0}{\phi_{s}-1} A^{k} B u(t-k)\right)\right]
$$

en particulier, $\quad x_{i}(t)=\bigoplus_{r=1}^{N}\left(A^{\phi}\right)_{i r} x_{r}\left(t-\phi_{s}\right) \oplus\left[\bigoplus_{k=0}^{\phi_{s}-1}\left(\underset{i^{\prime}=1}{\oplus}\left(A^{k} B\right)_{i i^{\prime}} u_{i^{\prime}}(t-k)\right)\right]$.

En tenant compte de (11), la contrainte (5) est satisfaite si les deux conditions suivantes sont vérifiées,

$$
\bigoplus_{r=1}^{N}\left(A^{\phi_{s}}\right)_{i r} x_{r}\left(t-\phi_{s}+\tau_{i j}^{\max }\right) \geq m_{i j} x_{j}(t),
$$

et

$$
\underset{k=0}{\phi_{s}-1}\left(\oplus_{i^{\prime}=1}^{m}\left(A^{k} B\right)_{i i} u_{i^{\prime}}\left(t-k+\tau_{i j}^{\max }\right)\right) \geq m_{i j} x_{j}(t) .
$$

Ensuite, nous prenons en considération l'inéquation (10), les deux inégalités suivantes

et

$$
\begin{aligned}
& \underset{r=1}{\oplus}\left[\left(\left(A^{\phi_{s}}\right)_{i r}-m_{i j}\right) x_{r}\left(t-\phi_{s}+\tau_{i j}^{\max }\right)\right] \geq\left(A^{\lambda_{s}} B\right)_{j s} u_{s}\left(t-\lambda_{s}\right), \\
& \underset{k=0}{\phi_{s}-1}\left[\bigoplus_{i^{\prime}=1}^{m}\left(\left(A^{k} B\right)_{i i^{\prime}}-m_{i j}\right) u_{i^{\prime}}\left(t-k+\tau_{i j}^{\max }\right)\right] \geq\left(A^{\lambda_{s}} B\right)_{j s} u_{s}\left(t-\lambda_{s}\right),
\end{aligned}
$$

impliquent la contrainte temporelle (5). 
Nous choisissons $\phi_{s}=\tau_{i j}^{\max }+\lambda_{s}+1$, et $u_{i^{\prime}}(t)=\varepsilon$ pour $i^{\prime} \neq s$, comme précisé dans le théorème, et compte tenu des conditions (v) et (vi), on voit clairement que la composante $u_{s}(t)$, donnée par l'équation

$$
u_{s}(t)=\bigoplus_{r=1}^{N}\left[\left(\left(A^{\phi}\right)_{i r}-\left(A^{\lambda_{s}} B\right)_{j s}-m_{i j}\right) x_{r}(t-1)\right],
$$

garantit le respect la contrainte temporelle (5).

Nous étendons ce résultat au cas où plusieurs contraintes doivent simultanément être assurées, dans l'esprit du théorème 3.

Corollaire 2 Le vecteur $u(t)$ qui est défini comme suit :

pour $l=1$ à $Z$,

$$
u_{l}(t)=\bigoplus_{z=1}^{Z} u_{z}(t)
$$

avec $u_{z}(t)$ la loi de commande calculée par le théorème 3 pour vérifier la $z^{\text {éme }}$ contrainte, et si $l \neq z$ pour $z=1$ à $Z, u_{l}(t)=\varepsilon$,

permet la satisfaction de toutes les $Z$ contraintes temporelles (9).

Démonstration La loi de commande $u_{z}(t)$ valide la $z^{\text {éme }}$ contrainte, si les conditions (v) et (vi) du théorème 3 sont satisfaites. Nous avons, pour $z=1$ à $Z$,

$$
u_{z}(t) \geq \underset{z=1}{Z} u_{z}(t)
$$

Il est donc clair, que la commande $u(t)=\oplus_{z=1}^{Z} u_{z}(t)$ assure le respect de toutes ses $Z$ contraintes temporelles.

\section{Application}

Nous nous sommes intéressés à un exemple réel de processus de production sous contrainte de temps. Ce processus fait partie d'une ligne de fabrication de tube de caoutchouc pour équipement automobile. Il est composé de convoyeurs en boucle, comme on peut le voir sur la Figure 5. Il comporte un poste de garnissage A, où sont fixées sur des palettes les pièces à traiter, un poste de dégarnissage $\mathrm{E}$ où celles-ci sont démontées, et un four (I O). Le four est constitué de deux parties, une zone de chauffe et une zone de refroidissement. C'est le temps de séjour des palettes dans la zone de chauffe qui est critique. 


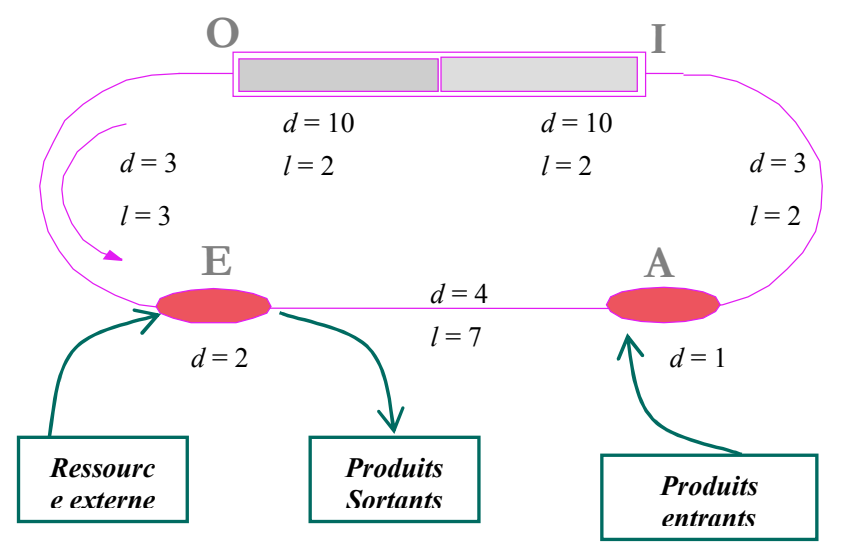

Figure 5 : Processus de production

Nous avons associé à ce processus un graphe d'événements temporisé donné par la Figure 6.

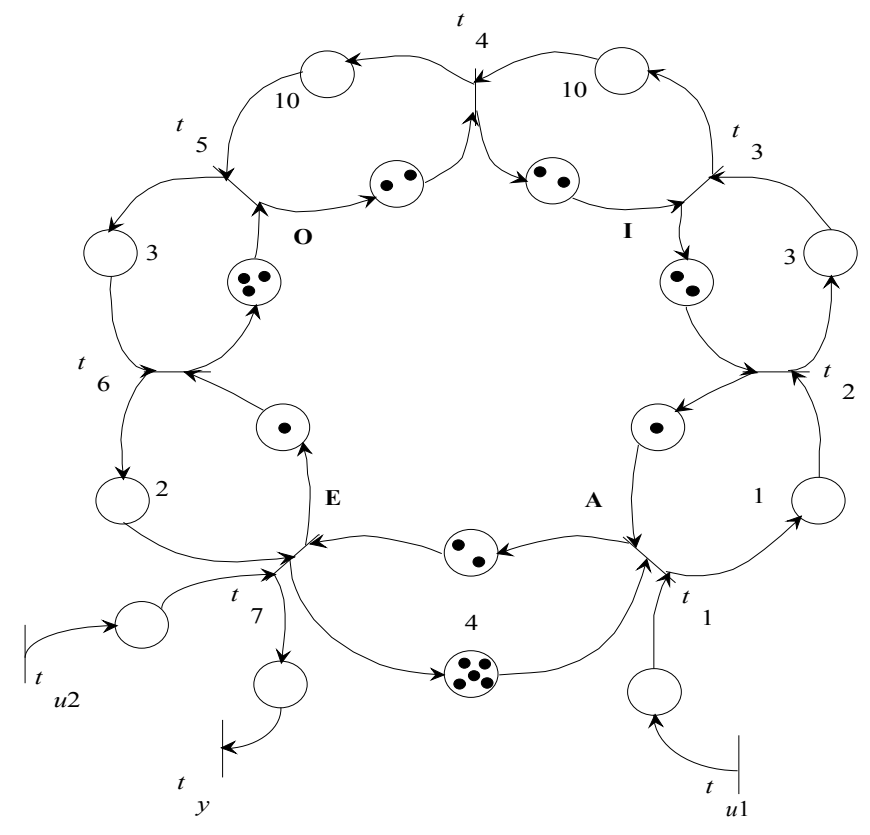

Figure 6 : Graphe d'événements temporisé associé

La transition d'entrée $u_{1}$ représente l'arrivée des pièces qui vont subir le traitement thermique et la transition $t_{7}$, qui joue le rôle que de sortie $y$, correspond à la sortie des pièces finies. Le franchissement de la transition $t_{1}$ correspond au 
début de l'opération de garnissage d'une palette, et $t_{2}$ à la fin de cette opération. Les durées des opérations sont indiquées sur les places correspondantes, par exemple, le transfert d'une palette du poste de garnissage à l'entrée du four (point I) dure 3 unités de temps. La transition $u_{2}$ est une entrée supplémentaire qui représente des moyens de transport des produits finis, on suppose qu'elle n'est pas contraignante au fonctionnement du système. La place $p_{34}$ modélise la zone chaude du four, sa temporisation qui correspond à la durée de cuisson des pièces, est déterminée, et vaut 10 unités de temps. Cette contrainte de temps se traduit par l'inéquation (12).

$$
x_{4}(t) \geq x_{3}(t-10),
$$

pour $t \geq 10$.

Nous allons alors déterminer une commande $u_{1}(t)$ qui garantit le respect de la contrainte temporelle (12). Pour cela, nous appliquons le théorème 1 . Pour écrire l'équation d'état du système et appliquer la formulation (4), nous allons étendre le graphe de la Figure 6 à ce que le graphe étendu contient que des places avec des temporisations inférieures ou égales à 1 . Nous définissons de nouvelles variables qui correspondent à des fonctions compteurs des transitions supplémentaires.

$x_{2 l}(t)=x_{2}(t-l)$ pour $l$ allant de 1 à $2, x_{3 l}(t)=x_{3}(t-l)$ pour $l$ allant de 1 à 9 ,

$x_{4 l}(t)=x_{4}(t-l)$ pour $l$ allant de 1 à $9, x_{5 l}(t)=x_{5}(t-l)$ pour $l$ allant de 1 à 2 ,

$x_{61}(t)=x_{6}(t-1)$ et $x_{7 l}(t)=x_{7}(t-l) \quad$ pour $l$ allant de 1 à 3.

La matrice d'état du graphe d'événement étendu est $A \in \overline{\mathbb{R}}_{\min }^{33 \times 33}$.

Nous avons pour cet exemple, $\tau_{i j}^{\max }=\tau_{43}^{\max }=10$ et $\lambda=4$, temporisation du chemin $\alpha$ qui relie les transitions $u_{1}$ et $t_{3}$. Le marquage initial de la place $p_{34}$ est $m_{i j}=m_{21}=e$ et le marquage du chemin $\alpha$ est $\left(A^{\lambda} B\right)_{j u}=\left(A^{4} B\right)_{31}=e$.

D'après le corollaire 1 , la loi de commande suivante :

$$
u_{1}(t)=\oplus_{r=1}^{33}\left(A^{15}\right)_{4 r} x_{r}(t-1),
$$

garantit le respect de la contrainte (12).

Nous calculons alors l'expression $x_{4}(t)=\stackrel{33}{\oplus}\left(A^{15}\right)_{4 r} x_{r}(t-15)$, qui est la fonction $\mathrm{du}$ compteur de la transition $t_{4}$ en fonction des autres variables retardées de 15 unités de temps. Après les calculs, nous avons abouti à la loi de commande suivante :

$$
u_{1}(t)=1 x_{1}(t-1) \oplus 3 x_{35}(t-1) \oplus 7 x_{48}(t-1) \oplus 4 x_{45}(t-1) \oplus 10 x_{51}(t-1) \oplus 5 x_{71}(t-1) .
$$

\section{Conclusion}

Nous avons défini deux conditions qui sont suffisantes pour l'existence d'une commande causale qui satisfait une contrainte temporelle donnée dans un graphe d'événements temporisé contrôlé. Nous avons appliqué cette méthode aux cas des 
graphes d'événements temporisés soumis à plusieurs contraintes de temps. Une généralisation de cette approche a été faite pour commander des graphes d'événements temporisés avec plusieurs entrées. Cette méthode a été illustrée sur un exemple d'un processus de production. Nous projetons des applications dans d'autres contextes, notamment pour la vérification et la validation des réseaux de télécommunication et les systèmes temps réels.

\section{Références}

[ALU 94] Alur, R. and D. Dill, A Theory of Timed Automata, Theoretical Computer Science (TCS), Vol. 126, No. 2, pp. 183-235, 1994.

[AMA 04] Amari S., I. Demongodin and J.J. Loiseau, Sizing, cycle time and plant control using dioid algebra, Chapter 6 in Supply Chain Optimisation, Series Applied Optimization, A. Dolgui J. Soldek and O. Zaikin (Eds.), Springer. pp. 71-85, 2004.

[AMA 05] Amari S., J.J. Loiseau, and I. Demongodin, Control of Temporal Constraints Based on Dioid Algebra for Timed Event Graphs, 19th IEEE International Parallel \& Distributed Processing Symposium (IPDPS), Colorado, 2005.

[BAC 92] Baccelli F., G. Cohen, G.-J. Olsder, and J.-P. Quadrat, Synchronization and Linearity: An algebra for Discrete Event Systems, Willey, 1992.

[BER 91] Berthomieu B. and M. Diaz, Modelling and verification of time dependant systems using time Petri nets, IEEE Transactions on Software Engineering, Vol. 17, No. 3, pp. 259-273, 1991.

[BON 01] Bonhomme P., P Aygalinc and S. Calvez, Systèmes à contraintes de temps : Validation, Evaluation et contrôle, MSR, Toulouse, 2001.

[GHE 91] Ghezzi C., D. Mandrioli, S. Morasca and M. Pezze, A unified high-level Petri net formalism for time critical systems, IEEE Transactions on Software Engineering, Vol. 17, No. 2, pp. 160-172, 1991.

[HOL 97] Holloway L.E., B.H. Krogh and A. Giua, A survey of Petri net methods for controlled discrete event systems, pp. 151-190, Kluwer Academic Publishers, Boston, 1997.

[LAH 04] Lahaye S., B. Cottenceau and A. Correïa, Commande des graphes d'événements temporisés avec contraintes de temps critique, In Proceedings Conf. Internationale Francophone d'Automatique (CIFA'04), Douz, Tunisie, Novembre 2004.

[MOO 94] Moody J., K. Yamalidou, M. Lemmon and P. Antsaklis, Feedback control of Petri nets based on place invariants, In Proceedings 34th IEEE Conference on Decision and Control (CDC'94), San Diego, 1994. 